\title{
El hejab (pañoleta islámica) en el Irán actual: una visión de las mujeres respecto a su uso
}

\author{
Masoumeh Velayati*
}

\begin{abstract}
This paper examines the different ways in which the hejab is perceived and used by Iranian women, emphasizing on the fact that the meanings given to hejab are contradictory and complex for veiled and unveiled women and it plays different roles in different contexts. The paper makes a comparison between the use of hejab before, during and after the Islamic revolution, and establishes a contrast between the literature written by muslim feminists, and the perception of some informants. It concludes that compulsory hejab is a patriarchal or fundamentalist tool used by men for controlling women, whereas voluntary hejab can be an emancipatory tool used by women for delivering themselves from patriarchal and cultural boundaries to legitimate their presence in public life whilst still retaining their cultural traditions.
\end{abstract}

Keywords: Islam, muslim feminism, contemporary Iran.

\section{Resumen}

El trabajo examina las diferentes formas en que las mujeres iraníes perciben y usan el hejab, enfatizando en el hecho de que los significados otorgados al mismo son complejos y contradictorios tanto para las mujeres que lo usan como para las que no, y juega papeles diferentes según los contextos. En el artículo se hace una comparación entre el uso del hejab antes, durante y después de la Revolución Islámica, y se contrasta la literatura escrita por feministas musulmanas con las percepciones de algunas informantes. El trabajo concluye que el hejab obligatorio es una herramienta patriarcal usada por los hombres para controlar a las mujeres, mientras que el hejab voluntario es una herramienta usada por las mujeres para liberarse a sí mismas de las ataduras patriarcales y culturales y para legitimar su presencia en la vida pública, al tiempo que conservan sus tradiciones culturales.

Palabras clave: Islam, feminismo musulmán, Irán contemporáneo

* Department of Politics, University of York, Correo-e: mv109@york.ac.uk 


\section{Introducción ${ }^{1}$}

Habiendo experimentado los procesos de modernización, secularización e industrialización durante más de medio siglo previo a la Revolución Islámica, Irán cambió el curso de su vida cotidiana bajo la gran influencia del Islam a través de una nueva interpretación. El paradigma elegido en este artículo para la interpretación de los procesos de descentramiento y pluralidad es el tema del hejab y sus muy complejos significados durante, antes y después de la revolución en Irán.

En el verano de 1998 llevé a cabo una investigación orientada a analizar si el hejab es opresivo para las mujeres iraníes como argumentan muchas feministas- o si las mujeres lo usan a su favor. En esta investigación participaron nueve mujeres, de las que, cinco eran amas de casa para quienes el hejab es una obligación religiosa; y cuatro eran mujeres que no usaban la pañoleta, una de ellas era empresaria, y dos estudiantes. Una de las estudiantes había decidido usarla o no usarla, dependiendo de las circunstancias. Estas mujeres eran originarias de diferentes lugares de Irán, vivían en el Reino Unido y provenían de diversos contextos sociales, tanto de familias religiosas y tradicionales, como de familias modernas orientadas al poder. Todas tenían una escolaridad de, por lo menos, nivel A.

El presente artículo está basado en una revisión bibliográfica, así como en entrevistas con estas nueve mujeres iraníes para examinar sus actitudes hacia el uso de la pañoleta y su carácter obligatorio en Irán. La revisión bibliográfica está basada principalmente en las discusiones de las feministas iraníes establecidas en Occidente, quienes tienen una variedad de argumentos acerca del papel del hejab en las vidas de las mujeres iraníes. Sin embargo, en general estos argumentos pueden dividirse en dos líneas fundamentales. Algunas de estas mujeres tienen una perspectiva muy negativa respecto al hejab. Para ellas, el hejab es el máximo símbolo de la dominación fundamentalista sobre las vidas de las mujeres, así como un símbolo de la opresión y subordinación de la mujer. Esto en lo que se refiere principalmente al movimiento de retorno al uso de la pañoleta entre las mujeres iraníes a finales de la década de los años setenta. Sin embargo, otras feministas examinan el tema del hejab en el interior de los contextos en que éste es adoptado. Para ilustrar los diferentes argumentos alrede-

${ }^{1}$ Traducción de Alma Mancilla. 
dor del tema del uso de la pañoleta en Irán, es esencial llevar a cabo una breve revisión de los principales cambios políticos que han tenido lugar en el Irán del siglo xx, y que han jugado un papel preponderante en la conformación del tema de la mujer.

\section{El tema de la mujer en la formación del moderno Estado iraní}

En el Irán del siglo xx, el tema de la mujer se ha situado como parte central de los discursos políticos debido a dos importantes revoluciones nacionalistas. En cada periodo, por la falta de alguna organización autónoma feminista y la consecuente carencia de una ideología feminista coherente, el tema de la mujer se volvió, más que una política radical de género, una parte esencial de los debates políticos alrededor de los cambios sociales. Por lo tanto, la primera decisión urgente para proteger al país de una situación de caos y dotarlo de fuerza en el poder político del Estado centralizado fue la introducción de la discusión acerca del uso o no uso obligatorio de la pañoleta.

En la primera mitad del siglo y a través de la imitación de los modelos occidentales, la esencia del movimiento nacionalista fue de naturaleza secular y el Islam fue asociado con el retroceso. Por lo tanto, la abolición de las prácticas tradicionales -como el matrimonio infantil, el uso de la pañoleta y la reclusión en nombre de la religión- se volvió tema de los debates políticos (Paidar, 1996: 52 y Afkhami, 1994: 6). Con el propósito de establecer una sociedad moderna basada en las reformas occidentales, Reza $\mathrm{Shah}^{2}$ planeó eliminar cualquier signo de tradicionalismo y 'retroceso'. Una de estas reformas consistió en la obligatoriedad de no utilizar la pañoleta, y fue instaurada el 8 de enero de 1936. La ley de abolición obligatoria del uso de la pañoleta y las políticas de género de Reza Shah fueron apoyadas por las mujeres y hombres de clase alta, así como por la recién creada clase media, debido a lo prometedoras que resultaban para la emancipación de la mujer (Paidar, 1996 y Ahmed, 1992). Ello, sin embargo, provocó la humillación, discriminación y reclusión de la mayoría de las mujeres de las clases populares (Hoodfar, 1993). Durante el mandato de su hijo, ${ }^{3}$ a pesar de la relajación en la obligatoriedad

\footnotetext{
${ }^{2}$ Fundador de la dinastía Pahlavi (1925-1979) que asciende al poder mediante un golpe militar en 1921, con el apoyo de Gran Bretaña, para asegurar los intereses británicos en Irán y evitar la penetración rusa en el sur de Asia (Ghods, 1989: 61-70).

${ }^{3}$ Segundo y último rey de la dinastía Pahlavi (1941-1979).
} 
de no usar la pañoleta, las políticas de discriminación evitaron el acceso de las mujeres con pañoleta a los empleos, debido a la ideología estatal oficial acerca de ésta (Hoodfar, 1993). Las desigualdades de género continuaron debido a la naturaleza limitada de las reformas para la mujer, así como a la desigual distribución de oportunidades.

Con excepción de una minoría de clase media alta, la mayoría de las mujeres fueron forzadas a cumplir sus obligaciones domésticas como parte esencial de sus vidas (Poya, 1992; Ashfar, 1989; Shahidian, 1997; Moghissi, 1993). La política de secularización, así como la existencia en la sociedad de creencias tradicionales, patriarcales y religiosas llevó a la mayoría de las mujeres a percibir el mensaje del Estado como contradictorio con respecto a su cultura tradicional. A mediados de la década de los setenta, con el ascenso del movimiento de oposición asociado con el Islam y su énfasis en los sentimientos antioccidentales y antiimperialistas, el hejab se volvió un símbolo de resistencia y solidaridad entre las mujeres (Paidar, 1996: 54-7 y Yeganeh, 1993: 7). Las mujeres más jóvenes y mejor educadas pertenecientes a la clase media tradicional, ambivalentes respecto a las reformas de Pahlavi, tenían un apasionado interés en un modelo de roles alternativos por medio del cual pudieran salvaguardar su presencia social y sus valores culturales indígenas y tradicionales. Para los activistas islámicos, el Occidente y sus modelos políticos y culturales eran y son aún considerados tanto opresivos, como culturalmente amenazadores.

Al criticar la explotación de la mujer como objeto sexual, resultado de la ciega imitación económica y cultural de Irán con respecto a Occidente, se introdujo el modelo islámico de la mujer, cuyo énfasis radica en el doble papel de las mujeres: como madres y revolucionarias (Yeganeh, 1993: 7). La crítica religiosa de la política de género de Pahlavi fue también apoyada por los izquierdistas seculares, quienes no tenían alternativa alguna a la desigualdad sexual y a las políticas de género de los Pahlavi (Shahidian, 1997 y Moghissi, 1993). Tanto para islamistas como para izquierdistas, una mujer aceptable en la sociedad iraní debía ser modesta, tanto en términos de expectativas sociales como de comportamiento y vestido (Najmabadi, 1991). Por otro lado, puesto que la ideología religiosa acerca de la mujer estaba basada en la libertad política prometida, la independencia cultural, la desigualdad económica y la justicia social, muchas mujeres renunciaron a las promesas de la modernización de acuerdo con lo que enten- 
dían desde su ideología islámica. Por lo tanto, un amplio grupo de mujeres, tanto seculares como religiosas, dio la bienvenida al nuevo modelo puritano de la mujer musulmana, basado en factores ideológicos y políticos, en oposición a los valores occidentales acerca de la mujer.

De este modo, a pesar de todas las consecuencias de la eliminación del hejab, este símbolo de 'retroceso' fue gradualmente adoptado por muchas mujeres educadas de clase media y media baja, como una protesta política o religiosa hacia las políticas de género adoptadas. El concepto de gharbzadegi (intoxicación occidental, westoxication), concepto compartido por todos los partidos, se volvió un medio útil de politizar el hejab (Najmabadi, 1991). El uso de la pañoleta, sin embargo, no significó retroceder a las prácticas tradicionales. Por el contrario, se trataba de un "regreso a la identidad cultural", algo nuevo por medio de lo cual las mujeres podían distinguirse tanto del modelo tradicional como del modelo occidental de mujer. Por esta razón, cuando adoptaban el hejab islámico, elegían una tela muy simple que no era ni tan restrictiva como el chador, ni tan moderna como la ropa occidental. Este nuevo hejab se volvió un símbolo del rechazo de la mujer hacia el concepto de "mujer consumista moderna", así como un símbolo de solidaridad para unificar a las mujeres de diferentes contextos sociales.

En la ideología islámica de género la maternidad era glorificada y las mujeres eran vistas como miembros invaluables de la familia. Así, después de la derrota del Shah y debido a la falta de una ideología feminista coherente, esta perspectiva ideológica fue percibida como de prosperidad para la mayoría de las mujeres, que se encontraban fundamentalmente marginadas en la era Pahlavi, a quienes se les otorgó un "papel 'central', 'fundamental' e 'invaluable', papel que nunca antes habían valorado" (Afshar, 1989: 119). Las nociones definidas por los varones -profundamente compartidas por algunas mujeres activistas- acerca de la debilidad biológica, emocional e intelectual de la mujer, y del papel destructivo de la sexualidad femenina, por un lado, y la debilidad del hombre frente al atractivo de la mujer, por el otro (Afshar, 1994), llevaron a esta noción de que la sexualidad de la mujer debía ser controlada y domesticada para prevenir su potencial poder seductor. A pesar de las protestas y demostraciones inmediatas por parte de las mujeres en contra de los conceptos fundamentalistas acerca de la imposición del hejab en el verano de 1980 , la mujer quedó sujeta a un rígido control. 
La imposición del código de vestimenta por parte del Estado islámico generó diferentes puntos de vista entre las feministas occidentales e iraníes, dentro y fuera de Irán. Para la mayor parte de quienes se oponen a ellos, la política de islamización y el hejab son los símbolos de la opresión de la mujer en Irán, debido a que han dado como resultado la exclusión de la mujer de algunos campos de estudio, la prohibición de la justicia, la imposición de reglas estrictas, etcétera. Para las mujeres iraníes que habían luchado por mucho tiempo para obtener un limitado nivel de igualdad y protección en la familia la política de islamización ha significado opresión y una derrota real. Así, para este sector de mujeres, el consentimiento de la mujer en la adopción del hejab es considerado como falsa conciencia por medio de la cual ellas juegan un papel muy importante en su propia 'desigualdad'.

Algunas otras feministas han percibido el otro lado de la moneda, y confirman los resultados opresivos del uso de la pañoleta, argumentando que los nuevos conceptos de mujer han jugado un papel emancipador en las vidas de muchas mujeres de clase baja y tradicional, en donde debido a sus códigos morales, éstos les han ofrecido una definición aceptable para el trabajo fuera del hogar. Así, dichas mujeres deben su presencia pública y su compromiso económico a la ideología islámica.

El fracaso del Estado islámico, debido a sus políticas contradictorias para responder a las promesas hechas a las mujeres respecto a la libertad política, la justicia social y la igualdad económica, motivaron a las feministas islámicas a oponerse a las políticas de género del Estado. Por lo tanto, desde finales de la década de los años ochenta al interior del establishment religioso y político, las feministas islámicas iraníes dieron inicio a su campaña autónoma contra las prácticas discriminatorias hacia la mujer en los ámbitos de educación, empleo, política y, de manera particular, la familia, mediante sus interpretaciones radicales centradas en la mujer respecto a la ley shari'a; y tuvieron éxito en eliminar algunas de las prácticas discriminatorias y en encabezar muchas otras reformas en favor de los intereses de la mujer. Sus campañas se orientaron, principalmente, contra los conceptos elaborados por los varones acerca de la mujer y la domesticidad, aparejados a sus interpretaciones de la ley islámica. La Ley de Protección a la Familia constituyó una plataforma para las feministas seculares durante el periodo del Shah, y fue abolida por su naturaleza no islámica inmediatamente después de la revolución. Pero las feministas islámicas lucharon por ella y lograron restaurarla nue- 
vamente en 1992; esta última no sólo perseguía los mismos objetivos, sino que incluso incorporaba algunas estipulaciones para otorgar mayor apoyo económico en las leyes de divorcio (MirHosseini, 1995 y 1996).

$\mathrm{Al}$ hacer referencia a los análisis de las feministas, pretendo dilucidar si las mujeres son embaucadas para usar la pañoleta, como argumenta la mayoría de estas feministas, o si la pañoleta es percibida de manera genuina como algo que responde a sus propios intereses. Pretendo observar si es posible emplear alguno de los argumentos de las feministas acerca del hejab y su carácter obligatorio en Irán, para arrojar luz acerca de las perspectivas de mis informantes y, a la inversa, si alguna de las perspectivas de mis informantes confirma o desafía estas posturas feministas.

\section{Puntos de vista de mis informantes acerca del hejab}

He clasificado mi análisis en cinco secciones: las impresiones sobre el hejab; el papel de la familia en la conformación de las ideas acerca del hejab; sus ventajas y desventajas; el énfasis de la República Islámica en el código de vestimenta de las mujeres; y la propia actitud de las mujeres hacia el hejab y su carácter obligatorio en el Irán actual.

De acuerdo con mis informantes, el hejab es una manifestación de diversos significados; en primer lugar el tipo de hejab indica la posición social de la mujer que lo usa; en segundo lugar, hace referencia a las relaciones de parentesco, puesto que la mujer musulmana usaría el hejab en presencia de na-mahrams $;^{4}$ en tercer lugar, hace referencia al tema de la sexualidad; en cuarto lugar, en los países no islámicos es un indicador de la identidad de quien lo usa; y lo más importante en el contexto iraní, demuestra la hegemonía de las políticas islámicas.

Aquellas informantes que usan pañoleta perciben el hejab como una obligación religiosa que todas las mujeres musulmanas deberían cumplir para demostrar un sentido interior de modestia al evitar todo tipo de pecados, particularmente el deseo sexual. Una de las mujeres dijo: "el hejab es una ley de Alá, nos guste o

${ }^{4}$ Mahram es, tanto para hombres como para mujeres, alguien con quien el matrimonio está prohibido usualmente debido al parentesco, ya sea por matrimonio o por relaciones ancestrales. Na-mahram es lo opuesto a mahram. Para una mujer, excepto para las mujeres o los jóvenes que no tienen aún deseos sexuales, los mahrams consanguíneos son el padre, los hermanos, los hijos, los sobrinos, los tíos y los abuelos; y los mahrams políticos son su esposo y su suegro. El resto serían na-mahrams, ante quienes debe cubrirse. 
no. Creo que el verdadero hejab proviene del corazón, y el hecho de cubrir el cuerpo es un reflejo material de las creencias interiores". Otra mujer dijo: "Si a ellas (a las mujeres que no usan la pañoleta) no les gusta usar el hejab, no deberían llamarse a sí mismas musulmanas." El puritanismo defendido por el Islam para evitar la expresión de los deseos sexuales de ambos sexos fuera del matrimonio fue mencionado a menudo por estas mujeres. Aunque las informantes que no usan la pañoleta no la consideran como un requisito religioso, sí la usan de manera voluntaria durante algunos eventos religiosos o en algunos contextos tradicionales. Cierta mujer que no usa la pañoleta mencionó: "Utilizo el hejab, si voy a una mezquita o si hay partes religiosas o ceremonias, o cuando lo necesito." Sin embargo, todas las informantes consideraron que el uso de una vestimenta apropiada es un valor para la mujer, pero no al grado en que el Islam lo recomienda.

A pesar de la influencia de diversos factores en la conformación de las ideas de mis informantes acerca del hejab, tanto las informantes que usan la pañoleta como las que no la usan, afirmaron haber seguido como modelo a sus madres respecto a usar o no el hejab, y esperaban que sus hijas, a su vez, las tomarían a ellas como modelo. Una mujer que usa la pañoleta afirmó:

Mi madre fue quien más influyó en mí. Ella constantemente nos aconsejaba prestar atención a nuestro hejab y a la matant (modestia). Ella solía decir que una chica es como una tela blanca. Una vez que una chica es acusada de no tomar en cuenta su castidad y su modestia, será como una tela blanca manchada, con una mancha que no puede limpiarse fácilmente. Contrario a lo que los occidentales puedan pensar acerca de que los padres, los hermanos o los esposos obligan a las mujeres a practicar el hejab, fue mi madre quien jugó el papel más significativo en mi decisión de utilizar la pañoleta.

El papel de los esposos no es tan determinante, aunque influyen en el uso del hejab en las mujeres, particularmente en los primeros años de matrimonio, debido a la importancia de la familia como una "institución sagrada" en la cultura iraní, así como al papel de la mujer en el mantenimiento de su dignidad. Esto se debe a que la mayoría de las parejas en Irán están cultural e ideológicamente establecidas antes del matrimonio y, por lo tanto, practicar o no practicar el hejab es a menudo un asunto que concierne a ambos. Una mujer que usa la pañoleta dijo:

Mi esposo está de acuerdo con el hejab. Una de nuestras condiciones de matrimonio fue que yo usaría hejab, no chador. Yo acepté esta condición, porque ya había tomado la decisión de hacerlo después de casarme. Por el contrario, rechacé a uno de mis pretendientes por exigirme 
usar chador. Si él me hubiera pedido que no usara pañoleta, tampoco lo habría aceptado. La razón es que existe una diferencia entre el hombre que exige usar el hejab, y el que exige que no se use en absoluto. El primero tiene algunos compromisos hacia la religión en su vida, mientras que el segundo puede no tenerlos para nada. Aún después de mi matrimonio yo siempre uso mi hejab de la forma en que a mí me interesa hacerlo, no como él me pide que lo haga. Durante un tiempo él quiso que cubriera mi cabello completo, pero yo cubro sólo una parte.

Por otro lado, una mujer que no usa pañoleta mencionó: "Si yo sigo a mi esposo, él me impondrá el uso de la pañoleta. Me parece que todos los hombres piensan de la misma manera."

Acerca de las ventajas del hejab, dos de las informantes mencionaron que no existe ventaja alguna en su práctica. Sin embargo, la mayor parte del resto de las informantes, tanto las que lo usan como las que no, percibe que el hejab, debido a su carácter obligatorio en Irán, es algo que está a favor de los propios intereses de la mujer, en términos de su papel en el respeto propio y en la autoprotección contra el acoso sexual de los hombres, la corrupción moral, los pecados, e incluso por la seguridad que proporciona en una situación de riesgo, tal como una mujer que no lo usa dijo:

...debido a que en Irán las mujeres son obligadas a usarlo, están seguras con el hejab, de lo contrario estarían en peligro. Las mujeres que se visten mal o que no usan la pañoleta pueden ser arrestadas o castigadas. Otra ventaja que le encuentro en nuestra sociedad es que el hejab es una forma de autoprotección contra el acoso de los hombres. Hasta donde recuerdo de mi juventud, cuando yo no usaba pañoleta en Irán, no me sentía segura cuando salía. Puesto que los hombres no son precisamente civilizados, siempre había abuso verbal y chistes groseros. Por las noches era aún peor, me parecía realmente amenazador. Si hubiera estado cubierta, probablemente me habría sentido mejor. Pero ahora desde que las mujeres en Irán se cubren, no creo que los hombres puedan acosarlas fácilmente.

Sin embargo, las mujeres que usan pañoleta se mostraron más preocupadas por las ventajas de ésta en los casos de consolidación de la unidad familiar y en la reducción de la tasa de divorcio, sacando a relucir la dedicación a los hijos, la salud y el bienestar de la familia y la sociedad. La familia ha sido siempre considerada por la mayoría en Irán como una institución clave para la salud, tanto de la sociedad como de los individuos. Desde esta perspectiva, la sociedad se compone de individuos, pero en forma de pequeños grupos llamados familias. Por lo tanto, a través del mejoramiento moral de las familias se garantizará la segu- 
ridad de la sociedad. Otra de las ventajas del hejab según las informantes que lo practican es que permite que las mujeres de familias religiosas y tradicionales tengan acceso a mayores niveles educativos, a empleos y a una mayor presencia social. En el periodo prerrevolucionario, debido a la hegemonía de la modernización, las mujeres que usaban la pañoleta no tenían un lugar y un estatus para expresarse en la sociedad. Sus familias tradicionales y religiosas tampoco les permitían ser parte activa de una sociedad llena de corrupción moral para ellas, así que eran prácticamente dependientes de sus parientes varones. Una de estas mujeres describe cómo:

En tiempos del Shah, debido a la corrupción existente en la sociedad, a las mujeres de las familias religiosas y conservadoras sus familias les impedían encontrar un trabajo o tener una mejor educación. Además, puesto que las mujeres que no usaban pañoleta tenían prioridad sobre las mujeres que la usaban, siempre había discriminación en la sociedad contra las mujeres que usaban pañoleta. Pero ahora, como todas las mujeres tienen que cubrirse y son casi lo mismo, no enfrentarán ninguna discriminación a condición de que tengan la misma preparación. Actualmente, las mujeres de las familias religiosas pueden ir a la universidad con chador, mientras que en aquella época eran humilladas, y esa era en sí misma otra razón para la falta de éxito de las mujeres que usaban pañoleta.

Otro de los aspectos que fue planteado por las mujeres que usan pañoleta es la unidad de las mujeres musulmanas y su respeto al Islam al practicar el hejab en el Occidente.

Acerca de las desventajas del hejab, el aspecto mencionado por ambos grupos fue la discriminación contra las mujeres que usan pañoleta en las sociedades occidentales. Las mujeres que la usan también mencionaron algunas dificultades prácticas, como la incomodidad del hejab durante los días calurosos del verano, para lo cual se negaron a usar la palabra "desventaja”, puesto que ellas creen que recibirán recompensas espirituales por las penurias que soporten por el Islam: "Alá ha previsto recompensas para aquellos que sean fuertes”, dijo una de las mujeres que usa pañoleta.

Sin embargo, las mujeres que no usan la pañoleta dieron diversas opiniones, como la restricción de los derechos y las libertades de la mujer; la falta de opciones para la mujer; el dictado de los hombres sobre las mujeres; la disminución del estatus de la mujer, la humillación de la posición de las mujeres en la sociedad; la opresión a la mujer; la desigualdad entre hombres y mujeres en términos del acceso a las oportunidades; y la incomo- 
didad. Las siguientes citas fueron expresadas por mujeres que no usan pañoleta:

Si estuviera en Irán, creo que me sentiría frustrada por estar cubierta todo el tiempo, particularmente cuando hiciera mucho calor. Algunas veces restringe la libertad de las mujeres, por ejemplo, cuando las mujeres quieren escalar montañas, la forma en que tienen que cubrirse lo hace difícil e incómodo, mientras que los hombres no tienen que hacerlo.

No sé qué tanto el hejab incidió en el bajo estatus social de la mujer en Irán. Pero creo que las diferencias entre hombres y mujeres se han tornado serias y el estatus de la mujer en la sociedad ha disminuido.

Me parece que ahora en Irán, puesto que se cubren la mayor parte del tiempo, las mujeres se ven disminuidas y son tratadas de manera desigual respecto a los hombres.

La imposición del hejab en Irán se percibió de acuerdo con cuatro perspectivas: el hejab como fenómeno religioso; como identidad nacional e independencia cultural; como un asunto de políticas sexuales; y como una demanda tanto política como religiosa. Las mujeres que usan pañoleta piensan que la revolución fue únicamente un movimiento religioso. Entonces, en un Estado islámico las exigencias del Islam deben ser respetadas tanto por el gobierno como por el pueblo. Una de ellas dijo:

Puesto que más del 90 por ciento de la población de Irán es musulmana, se trata de un país islámico, y el hejab es también una de las reglas islámicas mencionadas en el Corán. Es más, el pueblo de Irán se movilizó contra el Shah y hubo cerca de un millón de mártires para que pudiera haber un país islámico ideal. En una sociedad islámica ideal no debería permitirse a las mujeres comportarse de formas no islámicas. En la época del Shah, nuestra sociedad era casi el Occidente, teníamos bares y clubes y nuestras mujeres eran arrastradas a la corrupción, y todo lo que quedaba del Islam era el nombre. Así que hicimos la revolución, porque perseguíamos algo que fuera diferente del Occidente.

Sin embargo, para las mujeres que no usan pañoleta, la revolución fue un movimiento nacionalista y sus demandas fueron independientes de las religiosas. Piensan que durante la revolución, el papel de la mujer en la identificación de este nacionalismo cultural consistía en usar pañoleta. Con esta perspectiva, una mujer que no usa pañoleta explica, en una forma muy interesante, su propia experiencia con el hejab en el periodo revolucionario:

Nuestra profesora de literatura decía todo el tiempo que el Athey (el Occidente) estaba tomando nuestro petróleo gratis, mientras que en los pueblos de esta ciudad, en clima frío o en invierno, la gente no tenía petróleo para calentarse. Ella siempre estaba hablando de los pobres. Cuando el país se volvió contra el Shah, ella usaba hejab. En realidad 
ella siempre tenía mascadas extra, para dárselas a quien estuviera interesada. Yo nunca las usé, pero la mayoría de las alumnas sí.

Sin embargo, estas mujeres argumentan que la imposición del hejab es una forma sencilla de controlar a la mujer y de destruir sus derechos humanos y su libertad individual en el Estado Islámico.

Yo creo que es una forma de oprimir y controlar a la mujer. Por ejemplo, si una mujer no usa el hejab, no tendrá trabajo. De manera que es una forma de control.

Algunas mujeres perciben al hejab como un asunto tanto político como religioso, por lo que interpretaron la instauración del hejab obligatorio por parte del Estado Islámico en términos de autoridad, control de la mujer, e identificación y presentación de Irán como un verdadero país islámico frente al resto del mundo. Como menciona una mujer que no usa pañoleta:

Para demostrar verdaderamente el cambio. Para demostrar el estatus religioso de Irán a través de la mujer, y el hejab de la mujer era una manera de mostrarle al mundo que había una diferencia entre Irán como país islámico y los otros países islámicos. [...] Era (el hejab) y es una forma de control social, y las mujeres podían ser controladas fácilmente.

La política de occidentalización del régimen Pahlavi y la corrupción moral que estaba tan en boga fueron las principales razones dadas por mis informantes, tanto por las que practican el hejab como por las que no, para la creación de una revolución que persuadiera a la mujer y después a las autoridades para la adopción y la imposición del hejab. Valdría la pena citar un comentario de aquellas que participaron en la revolución. Al respecto, una de mis informantes enfatizó que:

Irán estaba en verdad perdiendo su cultura al irse al extremo. Yo era estudiante en la universidad y estaba convencida de que el Shah era un títere de los Estados Unidos. Había una enorme brecha entre ricos y pobres. Yo era joven y tenía mucha esperanza acerca del futuro de Irán y pensaba que si derrocábamos al Shah, tendríamos más libertad. Durante la revolución yo me cubría. Fue una pérdida cubrirme el cabello y el cuerpo, pero no el chador.

A pesar de la aceptación activa del hejab por parte de las mujeres durante la revolución, en tanto símbolo de regreso a la identidad cultural, ellas se oponían principalmente a su imposición por parte del Estado Islámico. En general, todas mis informantes sostenían que el hejab debería ser una cuestión privada y que no tenía nada 
que ver con la política. Respecto a la imposición del hejab a las mujeres iraníes, una mujer que sí lo practica dijo:

No puedo decir que se impuso de manera obligatoria, fue una sugerencia. Recuerdo que el líder dijo que 'es mejor para las mujeres cubrirse, y si no lo hacen, dejaré a la nación lidiar con ello' y así fue como se volvió obligatorio. Al día siguiente muchos hombres acosaron a las mujeres que no llevaban pañoleta, hubo mucho terror y la gente realmente se asustó, y al día siguiente de eso, las mujeres que antes no usaban pañoleta llevaban el hejab. Así fue como sucedió, fue sugerido y entonces las mujeres se vieron amenazadas.

El descontento de las mujeres que no usan pañoleta con respecto al hejab fue muy claro cuando describieron cómo se sintieron cuando lo usaron por primera vez. Una mujer explicó su propia experiencia de la siguiente manera:

Puesto que nadie en nuestra familia usaba el hejab y yo no estaba acostumbrada a hacerlo, no me gustaba. La primera vez que usé el hejab sentía que no podía escuchar nada y no me sentía cómoda con él. Pero todos me decían que la pañoleta me hacía ver más bonita.

Sin embargo, el haber crecido en familias religiosas fue una buena razón para que las mujeres que usaban pañoleta estuvieran de acuerdo con el código de vestimenta, ya que la introducción de este no era un fenómeno nuevo en sus vidas:

Puesto que yo vivía en una pequeña ciudad religiosa en donde la gente practicaba el hejab, su introducción obligatoria no fue algo nuevo. La única cosa que me parecía interesante, era el hecho de que todas teníamos que lucir igual en la escuela al usar un vestido largo, pantalones y una mascada.

Respecto a la propia actitud de las mujeres hacia el hejab y su imposición, muchas mujeres que usan pañoleta están, en general, de acuerdo con usarla. Una de estas mujeres, sin embargo, estaba sorprendida por su carácter obligatorio. Pese a que creía en el uso del hejab en tanto exigencia religiosa, no estaba de acuerdo con su carácter obligatorio, porque haría que las mujeres que fueran obligadas a usarlo en Irán estuvieran descontentas no sólo con el hejab, sino con el Islam en general. Aquellas que usaban la pañoleta y que se encontraban de acuerdo con su obligatoriedad, influenciadas por la ideología político-religiosa del Estado gobernante, creían que en lo que concierne a los decretos religiosos, no hay punto de discusión, y puesto que el uso de la pañoleta es un decreto religioso, debe ser acatado por todas las mujeres musulmanas. El hejab es tan importante para ellas que acusaron a las mujeres que no lo usaban de no ser creyentes. Además, el uso 
obligatorio de la pañoleta es legítimo para ellas porque es una "ley de Dios" mencionada en el Corán, así como una ley de la República Islámica. En su lógica, las mujeres iraníes deberían respetar a los mártires de la revolución y de la guerra Irán-Irak que fueron asesinados en nombre de la castidad de la mujer. Entonces, por medio del hejab, las mujeres demostrarían su respeto por la sangre de los mártires y de sus familias. Aquellas que ya se habían beneficiado de alguna manera de las oportunidades educativas y ocupacionales durante el periodo del Shah, y que esperaban mayores reformas orientadas al mejoramiento de las desigualdades de género se sintieron despojadas de poder por el código de vestimenta. Para ellas, la política de islamización dio como resultado otro tipo de discriminación contra las mujeres a quienes no les interesaba para nada el hejab. Así que, para dos de ellas, esto jugó un papel muy importante en su decisión de permanecer en el Reino Unido.

Por el contrario, las mujeres que usan pañoleta mencionaron con frecuencia la política de discriminación del régimen secular contra las mujeres que usaban la pañoleta, la atmósfera de corrupción de la sociedad, y la humillación de las mujeres que usaban pañoleta en el periodo del Shah. Una gran cantidad de mujeres discriminadas y alienadas, cuyo número no es pequeño, encontraron muchas más oportunidades de disfrutar de las ventajas educativas y ocupacionales, y se sintieron dotadas de mayor poder y más protegidas bajo las políticas de género de la República Islámica.

\section{Conclusión}

Al considerar los argumentos de las feministas y los puntos de vista de mis informantes, queda claro que el hejab tiene significados complejos y contradictorios para las mujeres que lo practican y para las que no lo hacen. Al comparar los movimientos de retorno al uso de la pañoleta en el Irán prerrevolucionario y en diversas sociedades como Egipto y Turquía, con el carácter obligatorio que tiene en el Irán posrevolucionario, encontré que el hejab porta diferentes mensajes en contextos diferentes. El hejab obligatorio es una herramienta patriarcal o fundamentalista empleada por los hombres para controlar a las mujeres, mientras que el hejab voluntario puede ser visto como una herramienta emancipadora empleada por las mujeres de alto nivel educativo para liberarse de las ataduras patriarcales y culturales, así como 
para legitimar su presencia en la vida pública y conservar sus tradiciones culturales.

Un análisis cuidadoso deja en claro que tanto las mujeres que usan la pañoleta como las que no la usan son movidas por el mismo impulso y persiguen las mismas metas a largo plazo, pero la falta de una conciencia feminista no les permite vislumbrar sus intereses comunes en las problemáticas de la mujer. En vez de ello, las diferencias ideológicas abren una enorme brecha entre las mujeres islámicas y las seculares, que les impide comprender que su objetivo principal es liberarse de cualquier forma de dominación patriarcal, ya sea ésta islámica o secular. La adopción del hejab por parte de las mujeres a finales de la década de los años setenta, así como su resistencia al hejab obligatorio en sus diversas formas, y la reinterpretación y relectura de la ley shari' $a$, indican el desafío de la mujer contra la dominación patriarcal sobre sus vidas.

Por lo tanto, el hejab en sí mismo no simboliza la opresión de todas las mujeres musulmanas iraníes, la mayoría de las cuales se han acostumbrado a vivir con ello. Para aquellas que ya habían aceptado el hejab como parte de su identidad religiosa y cultural indígena, el código de vestimenta en el periodo posrevolucionario ha dotado a la mujer de una nueva identidad bajo la protección de éste. El hejab en sí mismo tampoco opera a favor de los intereses de todas las mujeres iraníes, algunas de las cuales no definen ni condensan los decretos religiosos e Islámicos sólo en el hejab, y ven su imposición como un ataque contra sus derechos individuales. Pero al mismo tiempo, el hejab podría considerarse como un estado de violencia legitimado contra aquellas que no definen o no reducen los decretos religiosos e islámicos sólo al hejab, aunque muchas de ellas lo hagan recurriendo al uso no deseado de la pañoleta, entre ellas algunas profesionistas que no eran aceptadas o que no eran bien vistas por las mujeres durante la época prerrevolucionaria. Por lo tanto, lo que resulta importante es el contexto en el que el hejab se utiliza y la aplicación práctica que las mujeres hacen de él. 


\section{Bibliografía}

Afkhami, Mahnaz (1994), "Women in Post-Revolutionary Iran: A Feminist Perspective", in In the Eye of the Storm: Women in Post-revolutionary Iran, in M. Afkhami and Erika Friedl (eds.), I.B.Tauris, London and New York.

Afshar, H.(1989), "Women and Reproduction in Iran", in Nira Yuval-Davis and Floya Anthias (eds.), Woman - Nation State, MacMillan, London.

- (1994), Why Fundamentalism? Iranian Women and their Support for Islam, Working Paper, No. 2, Department of Politics, University of York, York.

Ahmed, Leila (1992), Women and Gender in Islam: Historical Roots of a Modern Debate, Yale University Press, New Haven.

Ghods, Reza (1989), Iran in the Twentieth Century: a Political History, Boulder Rienner Pub., London.

Hoodfar, H. (1993), “The Veil in Their Minds and On Our Heads: The Persistence of Colonial Images of Muslim Women", in Resources for Feminist Research: Colonialism, Imperialism and Gender, Vol. 22, Number 3/4.

Mir-Hosseini, Ziba (1995), "Stretching the Limits: A Feminist Reading of the Shari'a in Iran Today”, in M. Yamani (ed.), Feminism and Islam, Ithaca, London.

(1996), "Women and Politics in post-Khomeini Iran: Divorce, Ceiling and emerging feminist Voices", in H. Afshar (ed.), Women and Politics in the Third World, Routledge, London and New York.

Moghissi, Haideh (1993), "Women in the Resistance Movement in Iran", in Women in the Middle East, in H. Afshar (ed.), MacMillan Press, London. 
- (1994), "Women and Fundamentalism in Iran", in R. Lentin (ed.), Gender and catrophe, Zed Books, London and New York.

Najmabadi, Afsaneh (1991), "Hazards of Modernity and Morality: Women, State and Ideology in Contemporary Iran", in D. Kandiyoti (ed.), Women, Islam and the State, MacMillan Press, London.

Paidar, Parvin (1996), "Feminism and Islam in Iran”, in D. Kandiyoti (ed.), Gendering the Middle East: Emerging Perspectives, I.B Tauris, London and New York.

Poya, Maryam (1992), "Double Exile: Iranian Women and Islamic Fundamentalism", in Gita Sahgal and Nira Yuval-Davis (eds.), Refusing Holy Orders: Women and Fundamentalism in Britain, Virago Press, London.

Shahidian, Hamid (1997), "Women and Clandestine Politics in Iran, 1970-1985", in Feminist Studies, Vol. 23, No. 1 (Spring 1997), pp: 7- 43.

Yeganeh, Nahid (1993), "Women, Nationalism and Islam in Contemporary Political Discourse in Iran", in Feminist Review, No 44, Summer 1993, pp: 3- 19.

Enviado: 24 de octubre de 2001 Aceptado: 19 de noviembre de 2001 